\title{
Manual Therapy, Therapeutic Exercise, and HipTrac for Patients with Hip Osteoarthritis: A Case Series
}

John M Medeiros ${ }^{1 *}$ and Tony Rocklin²

${ }^{1}$ Pacific University, School of Physical Therapy, Hillsboro, USA

${ }^{2}$ Therapeutic Associates Physical Therapy, 837 SW 1st Avenue Suite 150, Portland, USA

\begin{abstract}
Background: Manual, long-axis hip traction has been used for centuries to treat pain and dysfunction associated with hip osteoarthritis $(\mathrm{OA})$. Now there is a way to replicate manual long-axis hip traction with a mechanical hip traction device (HipTrac) that can be used in the clinic and at home. The purpose of this case series is to describe a rehabilitation program that was used to treat two patients with hip OA.
\end{abstract}

Case description: Two patients were treated with manual therapy, therapeutic exercise, and HipTrac. The manual therapy and therapeutic exercise programs targeted impairments each patient presented with at each treatment session. The HipTrac, applied in the clinic and in each patient's home, was used for mobilizing the joint capsule and pain relief.

Outcomes: The primary outcome measures were the Care Connections Functional Index (CCFI), the Visual Analog Pain Scale (VAS), ROM, manual muscle tests, functional single leg squats and single leg dead lifts. Improvements in all outcome measures were observed for both patients.

Discussion: Clinically meaningful improvements in self-reported function and pain were described by both patients two years post-treatment. Both patients reported that they had greatly benefited from combining the individualized manual therapy and therapeutic techniques with the in-clinic and home use of the HipTrac.

Keywords: Osteoarthritis; Mechanical traction; Functional limitation; Hip stiffness; Gluteal muscle weakness; Hip traction

\section{Introduction}

Brackett stated in 1890 that "the value of traction in the treatment of the acute condition of hip disease has abundant evidence, both in its relief of the symptoms and in its influence on the course of the disease." Brackett credited Bradford and Conant for describing the position of traction, that is, when the hip is flexed and abducted. Brackett concluded that in "ordinary cases" when continual traction is used, distraction occurs and "this may happen even after disease has existed for some time." Brackett also noted that continual traction is beneficial for alleviating pain and for preventing the mechanical sequelae associated with excessive muscular irritability [1].

For decades, the first and most widely used manual therapy technique for hip joint pain has been long-axis hip traction. Many manual therapy techniques are important in the treatment of hip joint pathology; however, traction is one that can provide immediate pain relief while also working to improve general mobility in the long term. Based on the clinical findings obtained with manual therapy, including long-axis traction, and the need for continual traction as stated by Brackett, is there a way to couple these two concepts for improved patient care as it relates to hip joint pathology?

The purpose of this case series is to describe an augmented rehabilitation program wherein hip traction via the HipTrac (MedRock Inc., 101 SW Madison \#9262 Portland, OR 97207) was used by two patients with hip osteoarthritis (OA). In addition to using the HipTrac, the patients participated in an individually dosed and impairmentspecific manual therapy and therapeutic exercise program. The HipTrac, cleared by the FDA, is a home medical device that the patient can use to independently perform long-axis hip traction that replicates the manual technique performed in the clinic. It can be used in any degree of rotation and abduction as well as four levels of flexion $(0,10,20$, and
30 degrees.) The hip joint requires at least $400 \mathrm{~N}$ for distraction, and the HipTrac is able to produce forces well over $1000 \mathrm{~N}$. The HipTrac can also be used to perform lateral distraction in neutral, internal rotation, and external rotation as well as side-lying traction in extension. In this case series, the HipTrac was used only for supine longaxis traction in varying positions between close-packed and loose-packed hip positions. This is the first paper evaluating a multi-modal treatment approach to hip OA that allows the patient to receive long periods of hip traction at home as well as in the clinic.

\section{Background}

Within the last decade several authors have investigated the effects of manual therapy, including long-axis hip traction, as a component of the rehabilitation program for patients with hip OA. In a single-blind, randomized clinical trial of 109 patients with OA of the hip. Hoeksma et al. [2] reported statistically significant improvements in hip function [3] and pain (visual analog scale) in a group that received manual therapy (which included manual traction of the hip) versus a group that received exercise alone.

MacDonald et al. [4] described the outcomes from a series of seven patients with hip OA who were treated with manual therapy (including long-axis hip traction) and exercise. All patients exhibited reductions

*Corresponding author: John M. Medeiros, Pacific University, School of Physical Therapy, Hillsboro, USA, Tel: 5037058022; E-mail: medeiroj@pacificu.edu

Received: April 07, 2016; Accepted: April 21, 2016; Published: April 27, 2016

Citation: Medeiros JM, Rocklin T (2016) Manual Therapy, Therapeutic Exercise, and HipTrac for Patients with Hip Osteoarthritis: A Case Series. Physiother Rehabil 1: 108. doi: 10.4172/2573-0312.1000108

Copyright: () 2016 Medeiros JM, et al. This is an open-access article distributed under the terms of the Creative Commons Attribution License, which permits unrestricted use, distribution, and reproduction in any medium, provided the original author and source are credited. 
in pain (numeric pain rating scale), increases in passive hip range of motion, and improvements in function (Harris Hip Score [3]).

Vaarbakken and Ljunggren [5] compared the effectiveness of manual hip traction that was progressed to $800 \mathrm{~N}$ in 10 patients (experimental group) to a group $(\mathrm{n}=9)$ who received standard manual hip traction (force of the traction was unknown). Both groups received exercises, soft tissue techniques, and self-stretch procedures. Six out of the 10 subjects in the experimental group showed superior clinical post-treatment effects on the Hip Disability and Osteoarthritis Score [6] whereas none of the nine subjects in the control group showed as comparable improvement on the same outcome measure. The results suggest that higher known forces with manual hip traction are more effective in reducing self-rated hip disability after 12 weeks of treatment than the application of unknown manual traction forces provided by the clinician.

Wright et al. [7] retrospectively analyzed the data from 70 subjects who had participated in a randomized controlled trial. Forty-seven subjects were assigned to an exercise and manual therapy group (which included manual hip traction) and 23 subjects were assigned to a control group who received routine care offered by their general practitioner. Significant differences in the regression coefficients for the Global Rating of Change Scale [8] and the pain scale from the Western Ontario and McMaster Universities Osteoarthritis Index (WOMAC) [9] were found for the exercise/manual therapy group versus the control group.

Using the WOMAC as the primary outcome measure, Abbott et al. [10] allocated 206 adults with hip $(n=93)$ or knee $(n=113)$ OA to the following groups: usual care only $(n=51)$, usual care plus manual therapy $(n=54)$, usual care plus exercise therapy $(n=51)$, and usual care combined with exercise therapy and manual therapy $(n=50)$. For the participants with no joint replacement surgery during the trial (n $=162$ ), the authors reported statistically significant improvement in WOMAC scores for all three interventions; that is, manual physical therapy versus usual care, exercise therapy versus usual care, and the combined therapies versus usual care. The manual therapy group showed the greatest reductions in WOMAC scores of all groups overall and these reductions were still present one year later.

Using a randomized participant and assessor-blinded protocol trial with a 12-week intervention period, Bennell et al. [11] compared manual therapy, home exercises, education, and advice in 49 patients to a group of patients $(n=3)$ who received a sham treatment intervention.

All participants met the hip OA classification criteria of pain and radiographic changes set by the American College of Rheumatology [12]. The inclusion criteria were as follows: 50 years of age or older, pain in the hip or groin for more than 3 months, a Visual Analog Scale (VAS) score of 40 or higher on a $100 \mathrm{~mm}$ scale and at least moderate difficulty with activities of daily living. Major exclusion criteria included participation in physical therapy/chiropractic treatment in the past 6 months, prescribed exercises for the hip or lumbar spine in the past 6 months, current participation in a daily walking program for 30 minutes, or current participation in a regular structured exercise routine more than once weekly. The primary outcome measures were the VAS and the WOMAC. After 10 treatment sessions over 12 weeks, the investigators reported no significant differences between the treatment group and the sham treatment intervention. Based on the results of their study, the investigators concluded that "there is limited evidence supporting use of physical therapy for hip osteoarthritis."

\section{Case Description and Outcomes}

Each patient was informed that their physical therapy chart notes could be used in a publication or presentation. Each patient was informed that their identity would not be disclosed in a publication or presentation.

\section{Patient One}

Jill is a 50 -year-old female with a diagnosis by her orthopedic surgeon and radiographic evidence of moderate right hip OA. Her symptoms began 6.5 months ago and she describes her pain as sharp, dull, aching, throbbing, and constant in the groin and buttock regions. Her pain is aggravated by sitting, rising from sitting, walking, ascending/ descending stairs, and crossing legs. It is relieved by stretching, rest, and medication. She has been given the recommendation for total hip replacement at any time when she can no longer subjectively tolerate her pain and dysfunction. Jill's Care-Connections Functional Index (CCFI) score prior to receiving physical therapy was $52 \%$. A change of greater than $11 \%$ points has been reported as representing the MCID for the lower extremity [13]. Jill takes over-the-counter non-steroidal anti-inflammatory medications as needed. Jill rates her pain as 3 out of 10 on the Visual Analog Pain Scale (VAS). An MCID of $1.37 \mathrm{~cm}$ has been determined for the $10 \mathrm{~cm}$ VAS [14]. Jill's range of motion (ROM) on intake and discharge appears in Table 1.

Jill had the following positive signs on the right: Trendelenburg gait, FABER test, and a capsular pattern of restriction (defined here as loss of closed-pack position, FABERs, and flexion/internal rotation quadrant). She has increased hip pain with compression and decreased pain with traction. Jill's manual muscle test for hip abduction was 4-/5 on the right and $4+/ 5$ on the left. Jill could not perform a functional single leg squat with gluteal emphasis or a single leg dead lift without loss of balance, pelvic drop, or pain. The following goals and expected outcomes by time of discharge for her were as follows: independence with her home exercise program, pain rated as 1 out of 10 or less on the VAS, an increase in hip ROM (flexion to at least 1100, extension to at least 150 , internal rotation to at least 100 , and external rotation to at least 500), walking safely and independently all distances, and performing all normal work tasks with no limitations.

Jill received 17 physical therapy sessions over a span of 6 months with therapy provided $2 \mathrm{X}$ per week for 4 weeks, then $1 \mathrm{X}$ per week for 6 weeks, then 1X per month for 2 months, and finally 1 discharge visit 2 months later. Manual therapy in the clinic was focused on improving hip joint mobility and decreasing pain through a variety of techniques (Appendix A). Home and clinic therapeutic exercise programs focused on increasing lower extremity and lumbopelvic mobility, neuromuscular control, biomechanics, strength, flexibility and stabilization (Appendix B). HipTrac was initiated at home, after the eighth visit, to be used between visits and after discharge for pain-control and to supplement, reinforce, and further improve the hip mobility gains that she achieved with her clinical treatment (Appendix $\mathrm{C}$ - protocol)

\begin{tabular}{|c|c|c|c|c|}
\hline & \multicolumn{2}{|c|}{ Intake } & \multicolumn{2}{c|}{ Discharge } \\
\hline Hip ROM & Right & Left & Right & Left \\
\hline Flexion & 90 & 115 & 120 & 124 \\
\hline Extension & 9 & 15 & 20 & 25 \\
\hline Abduction & 28 & 40 & 35 & 45 \\
\hline Internal rotation (90 flexion) & 0 & 19 & 18 & 30 \\
\hline External Rotation (90 flexion) & 35 & 65 & 62 & 73 \\
\hline
\end{tabular}

Table 1: Jill's intake and discharge hip ROM in degrees. 
Jill's CCIF increased from $52 \%$ (intake score) to $86 \%$ (discharge score); this met the MCID of 11 points. Jill's VAS decreased from 3 (intake score) to 0.4 (discharge score); this met the MCID criteria of $1.37 \mathrm{~cm}$. Jill also reported that her global rate of change was $5 / 7$ at discharge. Between intake and discharge from physical therapy, Jill's ROM retest scores for her right hip increased by 300 for flexion, 110 for extension, 70 for abduction, 180 for internal rotation, and 270 for external rotation (Table 1 ).

When Jill was discharged, she reported that she rarely needed to take over-the-counter medications and was much more active now, participating in yoga twice per week in addition to her weekly home exercise program developed during treatment. Jill's hip abduction manual muscle test at discharge was $4+/ 5$ on the right as compared to 4-/5 at intake. In addition, Jill was able to perform functional single leg squats with gluteal emphasis and single leg dead lifts without loss of balance, pelvic drop, or pain greater than $1 / 10$ ( 2 sets of 10 of each) at discharge. Jill reported that she felt that she had greatly benefitted from home manual therapy using the HipTrac as well as her home exercise program. She verbalized understanding that her OA will progress and that consistent home manual therapy and exercise may continue to help her have less pain, increased mobility, and increased functionality. She reports her new goal is to more comfortably delay her surgery as long as possible. As of completion of this case series two years later, she has yet to have surgery and reports that she continues to maintain her higher level of function, reduced pain, and a more active lifestyle.

\section{Patient Two}

Travis is a very active 40 -year-old male with a diagnosis by his orthopedic surgeon and radiographic evidence of moderate left hip $\mathrm{OA}$ and left femoral acetabular impingement (FAI). He reports his symptoms began two years before with a gradual onset that he noticed while running. His chief complaint is a dull and constant ache in the left groin, thigh, and buttocks. Walking, stairs, and recreational sports such as running, skiing, cycling, hiking, and surfing aggravate Travis' symptoms; he reports that nothing relieves his symptoms. He has been given the recommendation for total hip replacement. Travis' CCIF score on intake was $90 \%$. A change of greater than or equal to 4 percentage points has been reported as representing the MCID for the lower extremity [14]. Travis takes over-the-counter non-steroidal antiinflammatory medications as needed. Travis rates his pain as 1.7 on the VAS. AN MCID of $0.4 \mathrm{~cm}$ or greater has been determined for the $10 \mathrm{~cm}$ VAS15. Travis' ROM on intake and discharge appears in Table 2.

At intake Travis had a positive left Trendelenburg, positive FABER test, and significant capsular restrictions. He had increased pain with compression and decreased pain with traction. His hip abduction muscle strength was $4 / 5$ on the left and $4+/ 5$ on the right. Travis could not perform a functional single leg squat with gluteal emphasis or a single leg dead lift without loss of balance, pelvic drop, or pain.

Expected goals and outcomes for Travis were as follows: home exercise program independence, pain rated as $1 / 10$ or less on the VAS, improved hip ROM (flexion to at least 1100 and internal rotation at 900 of hip flexion to at least 100), and participation in most of his recreational/sports activities with decreased symptoms less than $1 / 10$.

Travis received 15 physical therapy visits over a 5.5 month period with therapy provided $2 \mathrm{X}$ per week for 4 weeks, then $1 \mathrm{X}$ per week for 4 weeks, followed by 3 visits over the next 4 months. Manual therapy in the clinic focused on improving hip joint mobility and decreasing pain through a variety of techniques (Appendix A). Home and clinic

\begin{tabular}{|l|c|c|c|c|}
\hline & \multicolumn{2}{|c|}{ Intake } & \multicolumn{2}{c|}{ Discharge } \\
\hline Hip ROM & Right & Left & Right & Left \\
\hline Flexion & 115 & 85 & 120 & 112 \\
\hline Extension & 22 & 20 & 23 & 20 \\
\hline Abduction & 35 & 40 & 40 & 45 \\
\hline Internal rotation (90 flexion) & 28 & 0 & 27 & 14 \\
\hline External Rotation (90 flexion) & 40 & 50 & 45 & 51 \\
\hline
\end{tabular}

Table 2: Travis' intake and discharge hip rom in degrees.

therapeutic exercise programs focused on increasing lower extremity and lumbopelvic mobility, neuromuscular control, biomechanics, strength, flexibility, and stabilization (Appendix B). HipTrac was initiated at home, after the fourth visit, to be used between visits and after discharge for pain-control and to supplement, reinforce, and further improve the hip mobility gains that he achieved with his clinical treatment (Appendix C - protocol).

Travis' CCFI score increased from 90\% (intake) to $94 \%$ (discharge); this met the MCID of 4 points. Travis' VAS decreased from 1.7 (intake score) to 1 (discharge score); this met the MCID criteria of $0.4 \mathrm{~cm}$. His perceived global rate of change was 5/7 at discharge. Between intake and discharge from physical therapy, Travis' ROM retests scores for his left hip increased by 270 for flexion and 140 for internal rotation (Table 2). Travis' left hip abduction manual muscle test score at discharge was $4+/ 5$ as compared to $4 / 5$ at intake. In addition, Travis was able to perform 3 sets of 10 functional single leg squats and single leg dead lifts with proper technique and no pain over a 1/10 at discharge.

Near the end of Travis' physical therapy program, he reported that he had participated in a pain-free 62-mile bike ride. He also stated he was very happy to not only delay his total hip replacement but participate in more activities with less pain. He was able to return to surfing with some symptoms and could ride his bike daily for commuting without aggravating his hip. Against the advice of his medical team, he also returned to running 4-5 miles on trails three times per week with pain below a $2 / 10$. Because of his interest in regular participation in the high-level activities of surfing, running, and performance cycling, Travis reports that he has good days and days with some soreness; however, he now has improved mobility and strength in addition to pain management strategies to cope with any flare-ups. He reports that he can use the HipTrac and home exercise program to quickly decrease pain from increased activity and maintain hip mobility. He reported that he would not have been able to return to any of these activities nor delay hip surgery for the past two years if he had not used the HipTrac regularly at home.

\section{Discussion}

Providing individually dosed and impairment-specific manual therapy, therapeutic exercise, a home exercise program, and use of the HipTrac independently at home between visits and after discharge has increased the quality of life for these two patients. Hip traction has long been established as an effective therapy for patients with hip OA [1]. The most effective form of long-axis traction is when the distraction force is progressed [5]. The HipTrac allows the patient to receive prolonged and progressed distraction forces in the clinic and at home.

We have described a multi-modal rehabilitation program that produced subjective and objective results for these two patients. Our results are consistent with other authors $2,4,5,7,10$ who have reported benefits from manual therapy, exercise therapy, and a reinforcing home program. However, our findings are not supported by the work of Bennell et al. Differences between our case series and the Bennell 
et al study may be related to the following: 1) the dosage of manual therapy and therapeutic exercise provided; 2) the impairment-specific manual therapy techniques and therapeutic exercises provided to each individual patient or lack thereof and, 3) the activity level of the patients.

Regarding dosage, we spent more time with the patients than did Bennell et al. [11]. We believe that when treating such a complicated and varying pathology, a meaningful dose of manual therapy and therapeutic exercise cannot be properly applied in only 30 minutes and only one time per week. Some individuals may only need 30 minutes while others may require up to 60 minutes per session, with sessions being 1-2X per week for 4-6 weeks initially.

Regarding the manual therapy and exercise approach, our program was individualized for each patient whereas that of Bennell et al. used a semi-standardized approach to treatment. Random allocation of subjects into treatment and control groups is a very important component of a well-done study, as was the case with the Bennell et al. work. However, treatment for hip OA may need to be very specific to the individual's impairments, and providers may need to take special care to non-randomly categorize patients into the proper treatment protocol in order to show success. For example, clinical reasoning would discourage placing a patient with very good ROM into a manual therapy-emphasized category to increase ROM, just as we would not expect to place a patient with severe capsular restrictions into an exercise-only category. Treatment emphasis and categorization should depend on that individual's impairments.

In addition, all of the Bennell et al. subjects received only 2-3 different joint mobilization techniques: long-axis distraction in clinic and lateral distraction and/or inferior glide in hip flexion. Only $22 \%$ of the subjects in their active group also received joint mobilization in anterior glides for hip extension and external rotation, and $16 \%$ received posterior glides for internal rotation. It is well established that hip extension, internal rotation, and external rotation can be greatly limited with hip OA and are critical to specifically target in treatment when these limitations exist. In our case series, our two subjects received 8 different joint mobilization techniques, as needed, rather than only 2-5 techniques to specifically target each individual's impairments.

Also, Bennell et al. excluded patients under 50 years old as well as patients who could walk continuously for more than 30 minutes daily and those who participated in regular structured exercise more than once weekly. By excluding these individuals, Bennell et al. may only be studying individuals who are unmotivated to exercise/ improve, who are in too much pain or dysfunction to exercise, or who are fear-based individuals avoiding exercise. There is also a growing number of individuals younger than 50 years old that may benefit from treatment for hip OA earlier in the disease cycle. We believe that all individuals of all ages along the continuum of mild, moderate, and severe OA who are active and inactive more accurately represent those who need and may seek treatment for hip OA prior to becoming surgical candidates.

Evidence-informed practice takes into account what has been published in the literature, the experience of the clinician, and the goals of the patient. Consequently, success may need to be individually defined. There is no cure for hip OA and therefore providers cannot rid these patients of OA. The goals for most patients are to more comfortably avoid or delay surgery, improve mobility, decrease risk for co-morbidities due to inactivity related to their disease, decrease pain, and increase overall quality of life to engage in all of their social, occupational, and leisure activities. For some patients, making a change from a $7 / 10$ pain level and no participation in a regular exercise regimen to a $3 / 10$ pain level with consistent participation in an exercise regimen could equate to $100 \%$ success. For others, success could be to delay their hip replacement by 6 months for personal scheduling reasons while not having increased risk for hypertension or loss of blood glucose control due to inactivity. However, for all patients, we should not underestimate the significance of assisting them to become more active for at least 30 minutes per day to decrease the risk for heart disease, stroke, cancer, diabetes, depression, and other co-morbidities related to inactivity.

Total hip replacement is the gold standard of care once conservative measures have been exhausted and it is well documented that these individuals do very well after surgery in terms of functionality and quality of life. However, surgery is expensive, carries its own risks associated with being under general anesthesia, and will usually need to be repeated 15-20 years later on the same hip. From the point of view of the patient as well as that of the federal and private healthcare system, it is in the best interest to more comfortably delay this surgery as long as possible to decrease the overall healthcare utilization related to chronic pain and inactivity while improving the quality of the life for each individual.

We would like to emphasize the importance of evidence-based treatments including clinic and home manual therapy, therapeutic exercise, and patient education that can help each individual meet his or her specific goals. In this process we hope to discover which manual therapy techniques and therapeutic exercises, as well as which dosages of each, can help improve outcomes for individuals along the entire progressive continuum of hip OA and other hip joint pathologies.

Our two patients had joint mobility restrictions, muscle length deficits, muscle strength limitations, and insufficient muscle endurance/ coordination at intake. The two patients were gradually progressed to higher levels of clinical manual therapy, traction at home via HipTrac, therapeutic exercise, and soft-tissue stretch-and-release techniques such that the rehabilitation remained challenging. Our case study added home manual therapy, in the form of long-axis traction with HipTrac, as an additional benefit for the patients between visits and after discharge.

A limitation of any case series is that causality cannot be inferred from the data, especially with only two subjects. However, the findings can be used to inform clinical practice.

\section{Conclusion}

We have shown that providing manual therapy, exercise therapy, a home program, and home long-axis hip traction with the HipTrac provided clinically important improvements in pain and function for our two patients with OA of the hip. While not definitive, we also found objective and subjective feedback indicating that continuous and progressive hip traction, at home between visits and after discharge, has a valuable role to play in improving mobility and function while relieving pain in patients who have hip OA.

\section{References}

1. Brackett EG (1890) An experimental study of distraction of the hip-joint. Boston Med Surg J 122: 241-244.

2. Hoeksma HL, Dekker J, Ronday HK (2004) Comparison of manual therapy and exercise therapy in osteoarthritis of the hip: A randomized clinical trial. Arthritis Rheum 51: 722-729. 
Citation: Medeiros JM, Rocklin T (2016) Manual Therapy, Therapeutic Exercise, and HipTrac for Patients with Hip Osteoarthritis: A Case Series. Physiother Rehabil 1: 108. doi: 10.4172/2573-0312.1000108

3. Harris WH (1969) Traumatic arthritis of the hip after dislocation and acetabular fractures: Treatment by mold arthroplasty. An end-result study using a new method of result evaluation. J Bone Joint Surg Am 51: 737-755.

4. MacDonald CW, Whitman JM, Cleland JA, Smith M, Hoeksma HL (2006) Clinical outcomes following manual physical therapy and exercise for hip osteoarthritis: A case series. J Orthop Sports Phys Ther 36: 588-599.

5. Vaarbakken K, Ljunggren AE (2007) Superior effect of forceful compared with standard traction mobilization in hip disability? Adv Physiother 9: 117-128.

6. Klassbo M, Larsson E, Mannevik E (2003) Hip disability and Osteoarthritis Outcome Score: An extension of the Western Ontario and McMaster Universities Osteoarthritis Index. Scand J Rheum 32: 46-51.

7. Wright AA, Abbott JH, Baxter D, Cook C (2010) The ability of a sustained with-in session finding of pain reduction during traction to dictate improved outcomes from a manual therapy approach on patients with osteoarthritis of the hip. J Man Manip Ther 18: 166-172

8. Jaesehke R, Singer J, Guyatt GH (1989) Measurement of health status: Ascertaining the minimal clinically important difference. Control Clin Trials 10: 407-415.

9. Bellamy N (2002) WOMAC Osteoarthritis Index User Guide. Version V. Brisbane, Australia.
10. Abbott JH, Robertson MC Chapple C (2013) Manual therapy, exercise therapy, or both, in addition to usual care, for osteoarthritis of the hip or knee: A randomized controlled trial. 1: Clinical effectiveness. Osteoarthritis Cartilage 21: $525-534$.

11. Bennell KL, Egerton T, Martin J (2014) Effects of physical therapy on pain and function in patients with hip osteoarthritis: A randomized clinical trial. JAMA 311 1987-1997.

12. Altman R, Alarcon G, Appelrouth D (1991) The American College of rheumatology criteria for the classification and reporting of osteoarthritis of the hip. Arthritis Rheum 34: 505-514.

13. Hoekstra CJ, Deppeler DA, Rutt RA (2014) Criterion validity, reliability and clinical responsiveness of the CareConnections Functional Index. Physiother Theory Pract 30: 429-437.

14. Hawker GA, Main S, Kendzerska T, French M (2011) Measures of adult pain Visual Analog Scale for Pain (VAS Pain), Numeric Rating Scale for Pain (NRS Pain), McGill Pain Questionnaire (MPQ), Short-Form McGill Pain Questionnaire (SF-MPQ), Chronic Pain Grade Scale (CPGS), Short Form-36 Bodily Pain Scale (SF-36 BPS), and Measure of Intermittent and Constant Osteoarthritis Pain (ICOAP). Arthritis Care Res 63: S240-S252. 